\title{
Fenugreek with reduced bitterness prevents diet-induced metabolic disorders in rats
}

\author{
Etsuko Muraki ${ }^{1 *}$, Hiroshige Chiba ${ }^{1}$, Keiko Taketani ${ }^{2}$, Shohei Hoshino ${ }^{2}$, Nobuaki Tsuge ${ }^{2}$, Nobuyo Tsunoda ${ }^{1}$ \\ and Keizo Kasono ${ }^{1}$
}

\begin{abstract}
Background: Various therapeutic effects of fenugreek (Trigonella foenum-graecum L.) on metabolic disorders have been reported. However, the bitterness of fenugreek makes it hard for humans to eat sufficient doses of it for achieving therapeutic effects. Fenugreek contains bitter saponins such as protodioscin. Fenugreek with reduced bitterness (FRB) is prepared by treating fenugreek with beta-glucosidase. This study has been undertaken to evaluate the effects of FRB on metabolic disorders in rats.
\end{abstract}

Methods: Forty Sprague-Dawley rats were fed with high-fat high-sucrose (HFS) diet for 12 week to induce mild glucose and lipid disorders. Afterwards, the rats were divided into 5 groups. In the experiment 1 , each group $(n=8)$ was fed with HFS, or HFS containing $2.4 \%$ fenugreek, or HFS containing $1.2 \%, 2.4 \%$ and $4.8 \%$ FRB, respectively, for 12 week. In the experiment 2, we examined the effects of lower doses of FRB $(0.12 \%, 0.24 \%$ and $1.2 \%)$ under the same protocol ( $n=7$ in each groups).

Results: In the experiment 1, FRB dose-dependently reduced food intake, body weight gain, epididymal white adipose tissue (EWAT) and soleus muscle weight. FRB also lowered plasma and hepatic lipid levels and increased fecal lipid levels, both dose-dependently. The Plasma total cholesterol levels $(\mathrm{mmol} / \mathrm{L})$ in the three FRB and Ctrl groups were $1.58 \pm 0.09,1.45 \pm 0.05^{*}, 1.29 \pm 0.07^{*}$ and $2.00 \pm 0.18$, respectively $(* ; P<0.05$ vs. Ctrl). The Hepatic total cholesterol levels (mmol/g liver) were $0.116 \pm 0.011,0.112 \pm 0.006,0.099 \pm 0.007^{*}$ and $0.144 \pm 0.012$, respectively (*; $P<0.05$ vs. Ctrl). The calculated homeostasis model assessment as an index of insulin resistance (HOMA-IR) indicated $0.52 \pm 0.04^{*}, 0.47 \pm 0.06^{*}, 0.45 \pm 0.05^{*}$ and $1.10 \pm 0.16$, respectively $(* ; P<0.05$ vs. (trl). None of the FRB groups showed any adverse effect on the liver, kidney or hematological functions. In the experiment 2, no significant difference of food intake was observed, while the 1.2\% FRB group alone showed nearly the same effects on glucose and lipid metabolism as in the experiment 1.

Conclusions: Thus we have demonstrated that FRB (1.2 4.8\%) prevents diet-induced metabolic disorders such as insulin resistance, dyslipidemia and fatty liver.

Keywords: FRB, High-fat high-sucrose diet, Diet-induced metabolic disorders, Rats

\section{Background}

Recently, under the rapid economic development in advanced countries, lifestyle diseases including metabolic disorders are expanding. The principle of the basic therapeutic policy for these diseases consists of improvement of diet habits and enhancement of physical activities. However, it is difficult to control and change one's

\footnotetext{
*Correspondence: emuraki@ncnp.go.jp

${ }^{1}$ Department of Clinical Dietetics \& Human Nutrition, Faculty of

Pharmaceutical Sciences, Josai University, Saitama 350-0295, Japan

Full list of author information is available at the end of the article
}

established life styles. In such cases, functional foods with therapeutic effects on metabolic disorders are very helpful for the improvement of lifestyle diseases.

Various medicinal properties of fenugreek (Trigonella foenum-graecum L.) have been described earlier and its use in traditional Indian medicine is well known [1]. In recent years, fenugreek has been used in Western countries as a medicinal herb or as a spice. It has been reported that fenugreek reduced serum cholesterol level $[2,3]$, and improved blood glucose level and lipid metabolism in disease model animals of type 1 diabetes 
mellitus induced by such drug administration as alloxan $[4,5]$ and streptozotocin [6]. Recently, it has been reported that some active components of fenugreek, 4hydroxyisoleucine (4-OH-Ile) $[7,8]$ and galactomannan $[9,10]$, inhibited the elevation of blood glucose level and improved lipid metabolism in vivo. We have also reported that fenugreek reduced lipid levels in plasma and liver, leading to the improvement of insulin sensitivity in rats with metabolic disorders induced by a high-fat high-sucrose diet [11]. In our recent experiments, fenugreek dose-dependently inhibited the lipid accumulation in liver by increasing the excretion of lipid and bile acids in feces. An effective, safe and tolerable dose of fenugreek was about $2.5 \%(\mathrm{w} / \mathrm{w})$ [12]. However, the strong bitterness of fenugreek makes it difficult for humans to eat sufficient amount of fenugreek for the improvement of metabolic disorders. The main component of bitterness in fenugreek is protodioscin [13] Additional file 1]. So protodioscin was enzymatically converted into dioscin with no bitter taste.

The purpose of this study was to examine whether fenugreek with reduced bitterness (FRB) has such effects on diet-induced metabolic disorders as unmodified fenugreek, and to determine the most effective and tolerable dose of FRB.

\section{Methods}

\section{Materials}

Fenugreek and FRB were provided by House Foods (Chiba, Japan). FRB was made by adding cellulase enzyme (SPEZYME CP; Genencor, CA, USA) to fenugreek [14]. $82 \%$ of protodioscin was converted into dioscin in the FRB used for the experiment 1, and $96 \%$ of protodioscin was converted into dioscin in the FRB used for the experiment 2 . The enzyme also digested insoluble fiber fraction, hemicellulose and cellulose in fenugreek, and increased water-soluble fiber fractions in FRB. And the high molecular weight galactomannan (average molecular weight $2,000,000)$ in fenugreek was converted to the low molecular weight galactomannan (average molecular weight $500,000)$ in FRB. Thus FRB has a lower viscosity than fenugreek. As a result, FRB contains a larger amount of dioscin and soluble fiber fractions as compared with fenugreek itself.

\section{Animal care}

Three-week-old male, Sprague-Dawley rats were obtained from CLEA Japan, Inc. (Tokyo, Japan). The rats were housed individually in stainless steel wire-bottom cages in a room maintained at $22 \pm 2^{\circ} \mathrm{C}$ and $55 \pm 5 \%$ relative humidity with a $12 \mathrm{~h}$ cycle of light and dark. Tap water was given them freely throughout the experiment. The rats were fed a commercial diet (CE-2; CLEA Japan) for 1 week before proceeding to diet-induced metabolic disorders. Diets used in this study were high-fat highsucrose (HFS; lard $50 \% \mathrm{kcal}$, sucrose $25 \% \mathrm{kcal}$ ) diets based on the modified version of the AIN-93 G [15]. The rats were fed high-fat high-glucose (HFS) diet ad libitum for 12 week to induce metabolic disorders. In both experiments 1 and 2, the rats with diet-induced metabolic disorders were randomly divided into 5 groups of 7-8 rats each. Each experimental group was fed with either of the experimental diets shown in Table 1 for an additional 12 week period. After the 12 week, the rats were individually transferred into metabolic cages, and their feces were separately collected for 1 day. At the end of

Table 1 The diets ${ }^{1}$ of experiment 1 and 2

\begin{tabular}{|c|c|c|c|c|c|c|c|}
\hline \multirow[t]{2}{*}{ (g) } & \multirow[t]{2}{*}{ Ctrl } & \multirow[t]{2}{*}{ Fen } & \multicolumn{5}{|c|}{ FRB } \\
\hline & & & $0.12 \%$ & $0.24 \%$ & $1.2 \%$ & $2.4 \%$ & $4.8 \%$ \\
\hline $\operatorname{Lard}^{2}$ & 260.000 & 258.626 & 259.912 & 259.823 & 259.115 & 258.231 & 256.461 \\
\hline Milk casein ${ }^{2}$ & 200.000 & 193.205 & 199.653 & 199.306 & 196.529 & 193.059 & 186.118 \\
\hline Sucrose ${ }^{2}$ & 312.000 & 312.000 & 312.000 & 312.000 & 312.000 & 312.000 & 312.000 \\
\hline a-Corn starch ${ }^{2}$ & 32.487 & 32.487 & 32.487 & 32.487 & 32.487 & 32.487 & 32.487 \\
\hline$\beta$-Corn starch ${ }^{2}$ & 97.461 & 92.345 & 97.050 & 96.638 & 93.347 & 89.232 & 81.004 \\
\hline Vitamin mixture $^{3}$ & 10.000 & 10.000 & 10.000 & 10.000 & 10.000 & 10.000 & 10.000 \\
\hline Mineral mixture ${ }^{4}$ & 35.000 & 34.264 & 34.953 & 34.907 & 34.535 & 34.070 & 33.139 \\
\hline Cellulose powder ${ }^{2}$ & 50.000 & 40.045 & 49.695 & 49.389 & 46.947 & 43.893 & 37.787 \\
\hline L-Cystin ${ }^{5}$ & 3.000 & 3.000 & 3.000 & 3.000 & 3.000 & 3.000 & 3.000 \\
\hline t-Butylhydroquinone ${ }^{5}$ & 0.052 & 0.052 & 0.052 & 0.052 & 0.052 & 0.052 & 0.052 \\
\hline Fenugreek $^{6}$ & - & 23.976 & - & - & - & - & - \\
\hline $\mathrm{FRB}^{7}$ & - & - & 1.199 & 2.398 & 11.988 & 23.976 & 47.952 \\
\hline
\end{tabular}

Ctrl: Control group, Fen: Fenugreek group. 'Based on the AIN-93 G and modified. ${ }^{2,3,4}$ Purchased from Oriental Yeast Co.,Tokyo, Japan. ${ }^{3}$ Vitamin mixture was based on the AIN-93 formation, and added with tartaric acid choline. ${ }^{4}$ Mineral mixture was based on the AIN-93 G formation. ${ }^{5}$ Purchased from Wako Pure Chemical Industries, Osaka, Japan. ${ }^{6,7}$ Purchased from House Foods Corp., Somatech Center, Chiba, Japan. ${ }^{7}$ FRB: Fenugreek with reduced bitterness. 
the experiment, the rats were brought to rest under pentobarbital sodium (100 mg/kg, ip) anesthesia. Their blood samples were collected from the abdominal aorta, and the plasma was separated by means of centrifugation $\left(2,000 \times \mathrm{g}\right.$ for $20 \mathrm{~min}$ at $\left.4{ }^{\circ} \mathrm{C}\right)$ and stored at $-30^{\circ} \mathrm{C}$ until analyzed. Immediately after the collection of the blood samples, the livers, the solei and the epididymal white adipose tissues (EWAT) were excised, weighed and stored at $-30^{\circ} \mathrm{C}$ for further analyses.

The experimental protocol was approved by the Institutional Animal Care and Use Committee of Josai University.

\section{Experimental diets}

The experimental diets of the experiment 1: HFS (Ctrl group) as control, the HFS containing $2.4 \%$ fenugreek (Fen group), 1.2, 2.4 and 4.8\% FRB (Table 1).

The experimental diets of the experiment 2: HFS (Ctrl group) as control, the HFS containing $2.4 \%$ fenugreek (Fen group), 0.12, 0.24 and 1.2\% FRB.

Water-soluble dietary fiber (SDF) and insoluble dietary fiber (IDF) were measured by the Modified Prosky Method [16]. Fenugreek contains $9.4 \%(w / w)$ of SDF and $32.1 \%(\mathrm{w} / \mathrm{w})$ of IDF, whereas FRB in the experiment 1 and 2 contained $13.4 \%(\mathrm{w} / \mathrm{w})$ of SDF and $12.6 \%(\mathrm{w} / \mathrm{w})$ of IDF, and $20.8 \%(w / w)$ of SDF and $12.7 \%(w / w)$ of IDF, respectively.

\section{Oral glucose tolerance tests and intraperitoneal insulin tolerance tests}

At the10th wk, the rats were deprived of food for $12 \mathrm{~h}$, and afterwards they were submitted to an oral glucose tolerance test (OGTT: $2 \mathrm{~g} / \mathrm{kg}$ b.w. glucose). And at the 11th wk, an intraperitoneal insulin tolerance test (IPITT: $0.75 \mathrm{U} / \mathrm{kg}$ b.w. human regular insulin) was performed. We measured blood glucose concentrations by Ascensia $^{\mathrm{TM}}$ DexterZII (Bayer Medical, Tokyo, Japan) using tail blood samples at 0, 30, 60 and 120 min after the glucose administration, and at $0,15,30,60$ and $120 \mathrm{~min}$ after the insulin administration. Likewise, we measured plasma insulin concentrations by commercial kit (Rat Insulin ELISA KIT, Shibayagi, Co., Gunma, Japan) using separated tail plasma samples at 0, 30, 60 and $120 \mathrm{~min}$ after the glucose administration.

\section{Blood analysis}

Plasma triglyceride, total cholesterol, AST and ALT were measured by colorimetric slides using the Fuji Dri-Chem 3500 (Fujifilm Corp., Tokyo, Japan).

\section{Hepatic and fecal lipid concentrations}

The hepatic and fecal lipids were extracted in accordance with the method of Bligh and Dyer [17] and the method of Folch [18], respectively. Each extract was solubilized by Triton-X100 (Wako Pure Chemical Industries, Osaka, Japan), and the lipid concentrations were determined enzymatically using commercial kits (Triglycerides E-Test Wako, Cholesterol E-Test Wako, Total Bile Acid Test Wako; Wako Pure Chemical Industries).

\section{Statistical analysis}

All data are expressed as the mean \pm SEM. Statistical analyses were carried out by means of the Statistical Package for Social Sciences (SPSS12.0 J for Windows; SPSS Japan Inc., Tokyo, Japan). The effects of treatment were analyzed by the methods as follows. In the fivegroup comparison, the variances were calculated by Levene's test. When the variances were unequal among the groups, statistical comparisons were made by a nonparametric test, Kruskal-Wallis test, using post-hoc Mann-Whitney U tests with the Bonferroni correction. When the variances were equal among the groups, statistical comparisons were made by a parametric test, oneway ANOVA, using post-hoc Tukey HSD test. In the two-group comparison, the variances were calculated by Levene's test and statistical comparisons were made with $T$-test. In OGTT and IPITT, statistical comparisons were made by means of repeated ANOVA, using post-hoc Tukey HSD test. Differences were considered significant at $P<0.05$.

\section{Results}

\section{Experiment 1}

After 12 week of dietary treatment, the final body weight, the liver weight, the EWAT weight, the soleus muscle weight and the energy efficiency ratio (EER) were reduced by FRB dose-dependently (Table 2). Likewise, the plasma total cholesterol and the hepatic total cholesterol and triglyceride were reduced by FRB dosedependently, whereas no significant difference was found in the plasma triglyceride (Figure 1A-D). Futhermore, the fasting blood glucose and plasma insulin levels decreased significantly in all FRB groups as compared with the Ctrl and Fen groups. Therefore, homeostasis model assessment as an index of insulin resistance (HOMA-IR) decreased significantly in all FRB groups as compared with the Ctrl and Fen groups (Table 3). And in the $4.8 \%$ FRB group, the area under the curve (AUC) of glucose in OGTT was significantly reduced as compared with the Ctrl group (Figure 2B). Moreover, the plasma glucose level decreased remarkably in the $4.8 \%$ FRB group at $120 \mathrm{~min}$ in OGTT and IPITT as compared with the other groups (Figure 2A,C).

\section{Experiment 2}

After 12 week of dietary treatment, no significant differences were found in body weight gain between the FRB groups and the Ctrl group, whereas the EER, the EWAT 
Table 2 Effect of fenugreek on the body weight, energy intake, tissue weights and plasma parameters of experiment 1

\begin{tabular}{|c|c|c|c|c|c|c|c|c|c|c|c|c|c|c|c|}
\hline \multirow{3}{*}{ Total energy intake (kJ) } & \multirow{2}{*}{\multicolumn{3}{|c|}{ Ctrl }} & \multirow{2}{*}{\multicolumn{3}{|c|}{ Fen }} & \multicolumn{9}{|c|}{$\mathrm{FRB}^{6}$} \\
\hline & & & & & & & \multicolumn{3}{|c|}{$1.2 \%$} & \multicolumn{3}{|c|}{$2.4 \%$} & \multicolumn{3}{|c|}{$4.8 \%$} \\
\hline & 32,156 & \pm & $225^{\mathrm{a}}$ & 32,233 & \pm & $175^{a}$ & 32,017 & \pm & $177^{\mathrm{a}}$ & 28,789 & \pm & $377^{b}$ & 26,268 & \pm & $1,240^{b}$ \\
\hline Final body weight (g) & 754 & \pm & $22^{\mathrm{a}}$ & 748 & \pm & $16^{\mathrm{a}}$ & 714 & \pm & $9^{a}$ & 625 & \pm & $16^{\mathrm{b}}$ & 546 & \pm & $19^{c}$ \\
\hline $\mathrm{EER}^{1}(\mathrm{mg} / \mathrm{kJ})$ & 3.95 & \pm & $0.37^{\mathrm{a}}$ & 4.05 & \pm & $0.46^{\mathrm{a}}$ & 2.75 & \pm & $0.14^{\mathrm{a}}$ & 0.80 & \pm & $0.23^{\mathrm{b}}$ & -2.98 & \pm & $0.58^{c}$ \\
\hline Liver weight (g) & 24.2 & \pm & $0.9^{\mathrm{a}}$ & 23.2 & \pm & $1.3^{\mathrm{a}}$ & 21.5 & \pm & $0.8^{\mathrm{ab}}$ & 18.9 & \pm & $0.5^{\mathrm{bc}}$ & 16.6 & \pm & $0.5^{c}$ \\
\hline EWAT $^{2}$ weight (g) & 23.7 & \pm & $1.3^{\mathrm{a}}$ & 23.8 & \pm & $1.1^{\mathrm{a}}$ & 21.4 & \pm & $1.2^{\mathrm{a}}$ & 12.8 & \pm & $1.2^{\mathrm{b}}$ & 7.9 & \pm & $1.0^{c}$ \\
\hline Soleuse weight (g) & 0.19 & \pm & $0.01^{\mathrm{ab}}$ & 0.21 & \pm & $0.01^{\mathrm{a}}$ & 0.15 & \pm & $0.02^{\mathrm{bc}}$ & 0.13 & \pm & $0.01^{c}$ & 0.11 & \pm & $0.01^{c}$ \\
\hline Plasma $\mathrm{TG}^{3}$ (mmol/L) & 2.67 & \pm & 0.53 & 3.05 & \pm & 0.57 & 4.03 & \pm & 0.64 & 3.46 & \pm & 0.61 & 2.93 & \pm & 0.52 \\
\hline Plasma TC ${ }^{4}(\mathrm{mmol} / \mathrm{L})$ & 2.00 & \pm & 0.18 & 1.84 & \pm & 0.15 & 1.58 & \pm & 0.09 & 1.45 & \pm & $0.05^{*}$ & 1.29 & \pm & $0.07^{*}$ \\
\hline AST (U/L) & 65.3 & \pm & 5.4 & 53.1 & \pm & 2.6 & 52.3 & \pm & $2.6^{*}$ & 53.3 & \pm & $1.4^{*}$ & 56.0 & \pm & 3.2 \\
\hline $\operatorname{ALT}(U / L)$ & 22.5 & \pm & 3.3 & 18.9 & \pm & 1.3 & 29.6 & \pm & $2.2^{*}$ & 40.1 & \pm & $1.7^{*}$ & 51.8 & \pm & $3.7^{*}$ \\
\hline $\mathrm{AST} / \mathrm{ALT}^{5}$ & 1.13 & \pm & $0.09^{\mathrm{a}}$ & 1.05 & \pm & $0.09^{\mathrm{a}}$ & 0.65 & \pm & $0.02^{\mathrm{bc}}$ & 0.49 & \pm & $0.02^{c}$ & 0.04 & \pm & $0.03^{c}$ \\
\hline
\end{tabular}

Ctrl: Control group, Fen: Fenugreek group. ${ }^{1} \mathrm{EER}(\mathrm{mg} / \mathrm{kJ})=$ Body weight gain $(\mathrm{mg}) /$ Total food intake $(\mathrm{kJ}) .{ }^{2}$ EWAT: Epididymal white adipose tissue. ${ }^{3} \mathrm{TG}$ : Triglyceride. ${ }^{4} \mathrm{TC}$ : Total cholesterol. ${ }^{5}$ The relative values for Normal rats' data; AST/ALT $=$ AST (U/L)/ALT (U/L)/Normal rats' data. ${ }^{6} \mathrm{FRB}$ contains $82 \%$ of protodioscin converted to dioscin. Values are expressed as Mean \pm SEM for 8 rats. Mean without a common superscript letter is significantly different, $P<0.05$ (ANOVA). Asterisks indicate a difference from Ctrl: $P<0.05$ ( $T$-test).

weight, the plasma total cholesterol and the hepatic total cholesterol and triglyceride decreased significantly only in the $1.2 \%$ FRB group in comparison with the Ctrl group (Table 4 and Figure 3B-D). And the soleus muscle weight and the fecal total cholesterol increased significantly in the 1.2\% FRB group as compared with the Ctrl and Fen groups (Table 4, Figure 3E,F). Futhermore, the fasting blood glucose levels decreased significantly in the 1.2\% FRB group as compared with the Ctrl group (Table 5). And the AUC of glucose in the OGTT significantly decreased in the $1.2 \%$

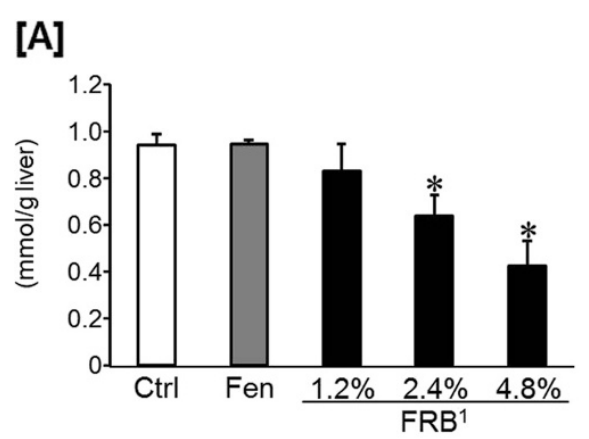

\section{[B]}

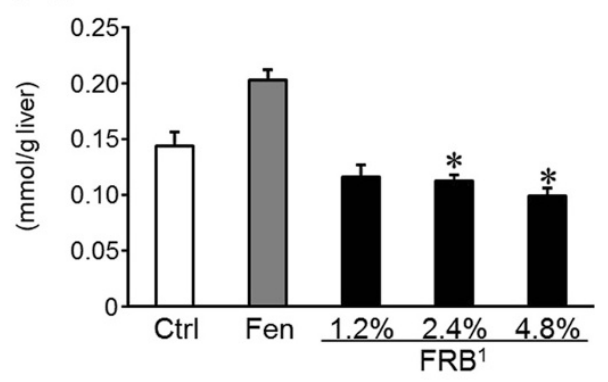

[C]

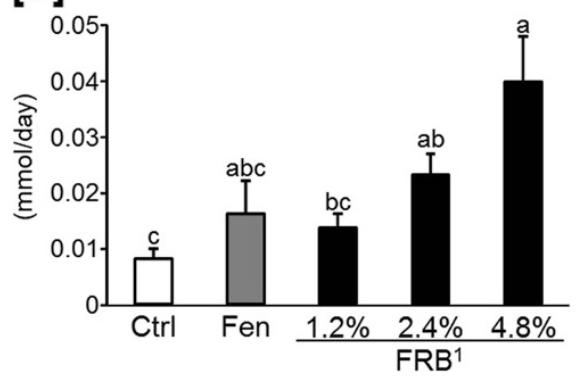

[D]

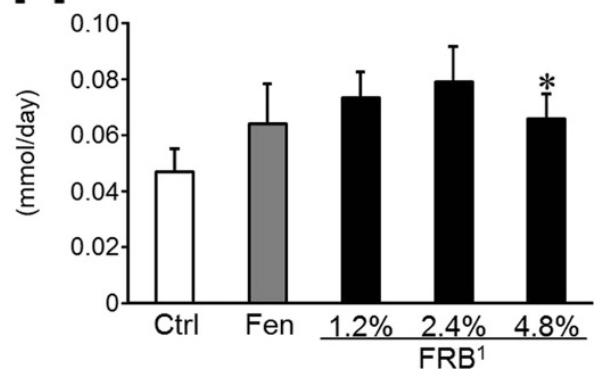

Figure 1 Effect of FRB on lipids in experiment 1. Hepatic triglyceride $[A]$ and total cholesterol $[B]$ levels. Fecal triglyceride $[C]$ and total cholesterol [D] levels. Ctrl: Control group, Fen: Fenugreek group. ${ }^{1}$ FRB contains $82 \%$ of protodioscin converted to dioscin. Values are expressed as Mean \pm SEM for 8 rats. Mean without a common superscript letter is significantly different, $P<0.05$ (ANOVA). Asterisks indicate a difference from Ctrl: $P<0.05$ (t-test). 
Table 3 The insulin resistance index of experiment 1

\begin{tabular}{|c|c|c|c|c|c|c|c|c|c|c|c|c|c|c|c|}
\hline \multirow{3}{*}{$\overline{\mathrm{FBG}^{\prime}(\mathrm{mmol} / \mathrm{L})}$} & \multirow[b]{3}{*}{5.79} & \multirow{2}{*}{\multicolumn{2}{|c|}{ Ctrl }} & \multirow{2}{*}{\multicolumn{3}{|c|}{ Fen }} & \multicolumn{9}{|c|}{$\mathrm{FRB}^{4}$} \\
\hline & & & & & & & \multicolumn{3}{|c|}{$1.2 \%$} & \multicolumn{3}{|c|}{$2.4 \%$} & \multicolumn{3}{|c|}{$4.8 \%$} \\
\hline & & \pm & 0.29 & 5.66 & \pm & 0.25 & 4.93 & \pm & $0.18^{*}$ & 4.78 & \pm & $0.19^{*}$ & 4.98 & \pm & $0.23^{*}$ \\
\hline $\mathrm{FPI}^{2}(\mathrm{nmol} / \mathrm{L})$ & 0.164 & \pm & $0.024^{a}$ & 0.142 & \pm & $0.009^{b}$ & 0.093 & \pm & $0.008^{\mathrm{ac}}$ & 0.086 & \pm & $0.010^{a c}$ & 0.081 & \pm & $0.010^{c}$ \\
\hline HOMA-IR ${ }^{3}$ & 1.10 & \pm & $0.16^{a}$ & 0.93 & \pm & $0.08^{\mathrm{a}}$ & 0.52 & \pm & $0.04^{b}$ & 0.47 & \pm & $0.06^{b}$ & 0.45 & \pm & $0.05^{\mathrm{b}}$ \\
\hline
\end{tabular}

Ctrl: Control group, Fen: Fenugreek group. ${ }^{1}$ FBG: Fasting blood glucose. ${ }^{2}$ FPI: Fasting plasma insulin. ${ }^{3}$ Relative values of (fasting blood glucose $\times$ fasting blood insulin) to the mean value of $\mathrm{Ctrl}$ group. ${ }^{4} \mathrm{FRB}$ contains $82 \%$ of protodioscin converted to dioscin. Values are expressed as Mean \pm SEM for 8 rats. Mean without a common superscript letter is significantly different, $P<0.05$ (ANOVA). Asterisks indicate a difference from Ctrl: $P<0.05$ ( $T$-test).

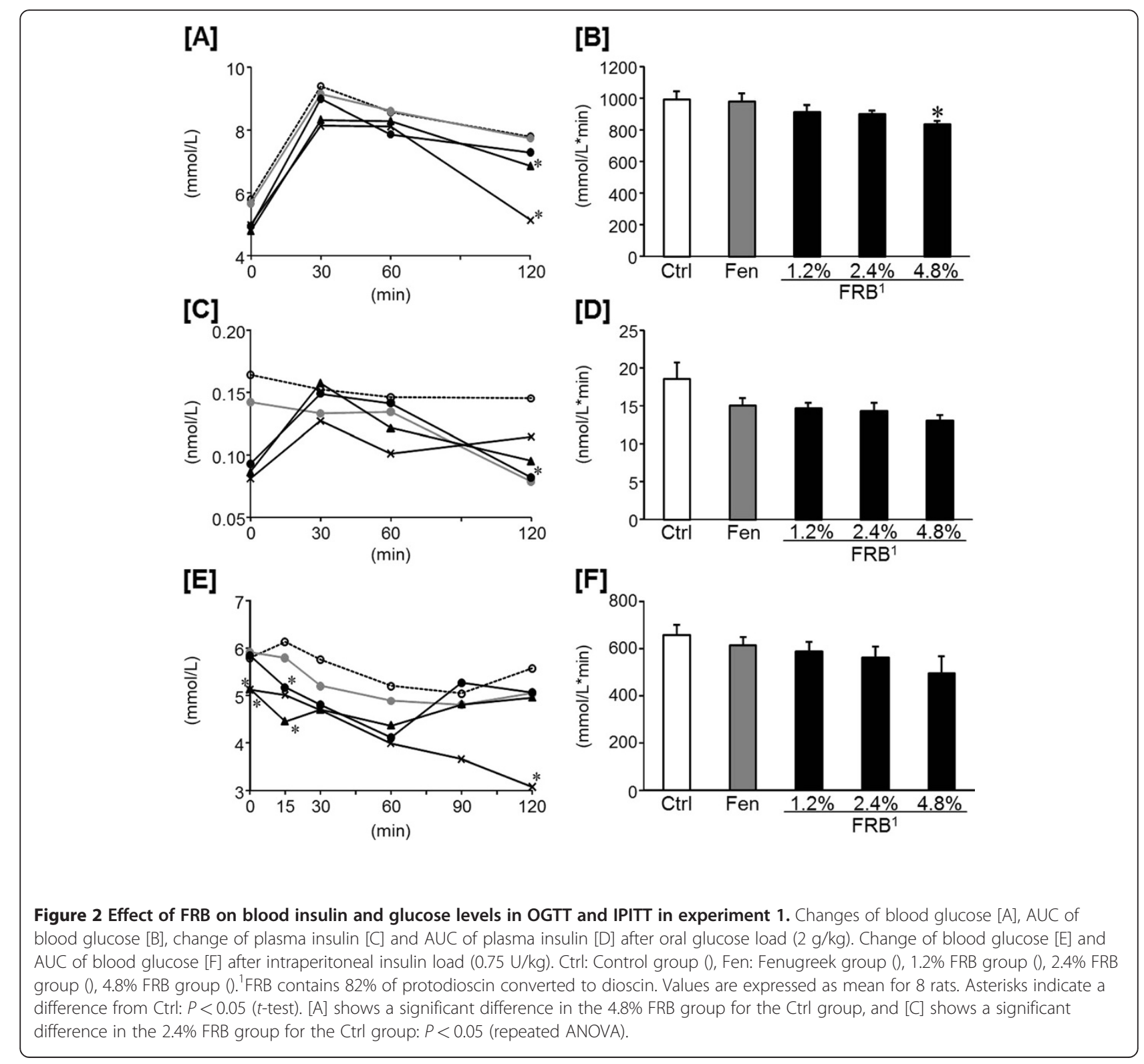


Table 4 Effect of fenugreek on the body weight, energy intake, tissue weights and plasma parameters of experiment 2

\begin{tabular}{|c|c|c|c|c|c|c|c|c|c|c|c|c|c|c|c|}
\hline \multirow{3}{*}{ Total energy intake (kJ) } & \multicolumn{3}{|c|}{$\mathrm{Ctrl}$} & \multirow{2}{*}{\multicolumn{3}{|c|}{ Fen }} & \multicolumn{9}{|c|}{$\mathrm{FRB}^{6}$} \\
\hline & \multirow[b]{2}{*}{34,686} & \multirow[b]{2}{*}{ \pm} & \multirow[b]{2}{*}{363} & & & & \multicolumn{3}{|c|}{$0.12 \%$} & \multicolumn{3}{|c|}{$0.24 \%$} & \multicolumn{3}{|c|}{$1.2 \%$} \\
\hline & & & & 35,076 & \pm & 184 & 35,332 & \pm & $42^{*}$ & 35,119 & \pm & $196^{*}$ & 35,319 & \pm & 40 \\
\hline Final body weight (g) & 757 & \pm & $21^{\mathrm{ab}}$ & 792 & \pm & $13^{a}$ & 789 & \pm & $18^{\mathrm{ab}}$ & 773 & \pm & $16^{\mathrm{ab}}$ & 717 & \pm & $14^{\mathrm{b}}$ \\
\hline $\mathrm{EER}^{1}(\mathrm{mg} / \mathrm{kJ})$ & 1.54 & \pm & 0.32 & 2.86 & \pm & 0.31 & 2.51 & \pm & 0.46 & 2.38 & \pm & 0.21 & 0.39 & \pm & $0.52^{*}$ \\
\hline Liver weight (g) & 21.3 & \pm & 0.9 & 21.3 & \pm & 1.1 & 21.6 & \pm & 0.7 & 21.8 & \pm & 1.4 & 19.3 & \pm & 0.6 \\
\hline EWAT $^{2}$ weight (g) & 21.0 & \pm & 0.6 & 23.6 & \pm & 1.4 & 22.7 & \pm & 1.6 & 20.3 & \pm & 1.2 & 15.7 & \pm & $1.5^{*}$ \\
\hline Soleuse weight (g) & 0.40 & \pm & 0.02 & 0.44 & \pm & 0.02 & 0.51 & \pm & 0.08 & 0.51 & \pm & 0.07 & 0.54 & \pm & $0.06^{*}$ \\
\hline Plasma $\mathrm{TG}^{3}(\mathrm{mmol} / \mathrm{L})$ & 1.34 & \pm & 0.24 & 1.73 & \pm & 0.36 & 1.49 & \pm & 0.34 & 1.43 & \pm & 0.37 & 1.27 & \pm & 0.26 \\
\hline Plasma TC $C^{4}(\mathrm{mmol} / \mathrm{L})$ & 1.95 & \pm & 0.11 & 1.80 & \pm & 0.05 & 1.81 & \pm & 0.12 & 1.76 & \pm & 0.15 & 1.60 & \pm & $0.09^{*}$ \\
\hline AST (U/L) & 58.8 & \pm & 4.1 & 56.5 & \pm & 3.5 & 52.0 & \pm & 2.9 & 57.0 & \pm & 4.5 & 52.4 & \pm & 1.4 \\
\hline $\operatorname{ALT}(U / L)$ & 19.6 & \pm & 2.0 & 21.1 & \pm & 2.5 & 18.3 & \pm & 1.8 & 20.3 & \pm & 3.5 & 25.0 & \pm & 4.0 \\
\hline $\mathrm{AST} / \mathrm{ALT}^{5}$ & 1.29 & \pm & 0.18 & 1.13 & \pm & 0.07 & 1.18 & \pm & 0.08 & 1.29 & \pm & 0.21 & 0.95 & \pm & 0.11 \\
\hline
\end{tabular}

Ctrl: Control group, Fen: Fenugreek group. ${ }^{1} \mathrm{EER}(\mathrm{mg} / \mathrm{kJ})=$ Body weight gain $(\mathrm{mg}) / T$ total food intake $(\mathrm{kJ}) .{ }^{2} \mathrm{EWAT}$ : Epididymal white adipose tissue. ${ }^{3} \mathrm{TG}$ : Triglyceride. ${ }^{4} \mathrm{TC}$ : Total cholesterol. ${ }^{5}$ The relative values for Normal rats' data; AST/ALT = AST (U/L)/ALT (U/L)/Normal rats' data. ${ }^{6} \mathrm{FRB}$ contains $96 \%$ of protodioscin converted to dioscin. Values are expressed as Mean \pm SEM for 7-8 rats. Mean without a common superscript letter is significantly different, $P<0.05$ (ANOVA). Asterisks indicate a difference from Ctrl: $P<0.05$ ( $t$-test)

FRB group as compared with the Ctrl group, whereas the AUC of glucose in the IPTT significantly decreased in the $0.24 \%$ FRB group as compared with the Ctrl group (Figure 4B, F). Moreover, in the OGTT, the plasma glucose levels in the $1.2 \%$ FRB group were significantly lower at 30 and 120 min than those in the Ctrl group (Figure 4A). And in the IPITT, the plasma glucose levels in the $0.24 \%$ FRB group decreased promptly after the insulin administration (Figure 4E).

\section{Discussion}

Our previous study showed that the effective dose of fenugreek was around $2.4 \%(\mathrm{w} / \mathrm{w})$ [12]. The present study has shown that the effective dose of FRB is lower than that of fenugreek.

The previous study demonstrated that fiber sources providing predominantly IDF, such as corn bran, cellulose and wheat bran, have little effect on plasma and liver cholesterol levels. On the other hand, fiber sources containing a mixture of both SDF and IDF, such as soybean and oat bran, have intermediate effects on plasma and liver cholesterol levels [19]. SDF may be fermentable in the large intestine by the action of colonic bacteria producing short chain fatty acids such as butyrate, propionate, acetate etc. [20], which can act to reduce cholesterol synthesis [21]. And the major functions of IDF fractions can be attributed to their passive water holding capacity and non-digestibility, which may help to increase the bulk and shortens the transit time of stool through the intestinal tract [22]. However, IDF showed no effect on cholesterol absorption. The increase of SDF fraction in FRB might induce remarkable lowering effects on plasma and liver cholesterol concentrations [23].

On the other hand, many reports have been published, and demonstrated that diosgenin (aglycon of protodioscin and dioscin) had effects on glucose and lipid metabolism. Diosgenin raises the peroxisome proliferator-activated receptor $\gamma$ (PPAR $\gamma$ ) level in WAT, and promotes adipocyte differentiation and size reduction of adipocytes. As a result, the secretion of monocyte chemoattractant protein-1 (MCP-1) in adipocytes is suppressed, whereas the secretion of adiponectin is promoted, and the inflammation in adipose tissue is inhibited [13,24]. Therefore, diosgenin is also a candidate component to be contained in fenugreek for the improvement of insulin sensitivity. In addition, saponins, such as diosgenin, form large micelles from bile acid and saponin molecules in the small intestine, and

Table 5 The insulin resistance index of experiment 2

\begin{tabular}{|c|c|c|c|c|c|c|c|c|c|c|c|c|c|c|c|}
\hline \multirow{3}{*}{$\overline{\mathrm{FBG}^{1}(\mathrm{mmol} / \mathrm{L})}$} & \multirow{2}{*}{\multicolumn{3}{|c|}{ Ctrl }} & \multirow{2}{*}{\multicolumn{3}{|c|}{ Fen }} & \multicolumn{9}{|c|}{$\mathrm{FRB}^{4}$} \\
\hline & & & & & & & \multicolumn{3}{|c|}{$0.12 \%$} & \multicolumn{3}{|c|}{$0.24 \%$} & \multicolumn{3}{|c|}{$1.2 \%$} \\
\hline & 5.70 & \pm & 0.24 & 5.93 & \pm & 0.40 & 5.69 & \pm & 0.30 & 5.21 & \pm & 0.22 & 4.95 & \pm & $0.25^{*}$ \\
\hline $\mathrm{FPl}^{2}(\mathrm{nmol} / \mathrm{L})$ & 0.178 & \pm & 0.045 & 0.235 & \pm & 0.052 & 0.176 & \pm & 0.029 & 0.368 & \pm & 0.095 & 0.165 & \pm & 0.018 \\
\hline HOMA-IR ${ }^{3}$ & 1.00 & \pm & 0.24 & 1.47 & \pm & 0.39 & 0.97 & \pm & 0.12 & 1.88 & \pm & $0.51^{*}$ & 0.81 & \pm & 0.08 \\
\hline
\end{tabular}

Ctrl: Control group, Fen: Fenugreek group. ${ }^{1} \mathrm{FBG}$ : Fasting blood glucose. ${ }^{2} \mathrm{FPI}$ : Fasting plasma insulin. ${ }^{3}$ Relative values of (fasting blood glucose $\times$ fasting blood insulin) to the mean value of $\mathrm{Ctrl}$ group. ${ }^{4} \mathrm{FRB}$ contains $96 \%$ of protodioscin converted to dioscin. Values are expressed as Mean \pm SEM for $7-8$ rats. Mean without a common superscript letter is significantly different, $P<0.05$ (ANOVA). Asterisks indicate a difference from Ctrl: $P<0.05$ ( $T$-test). 


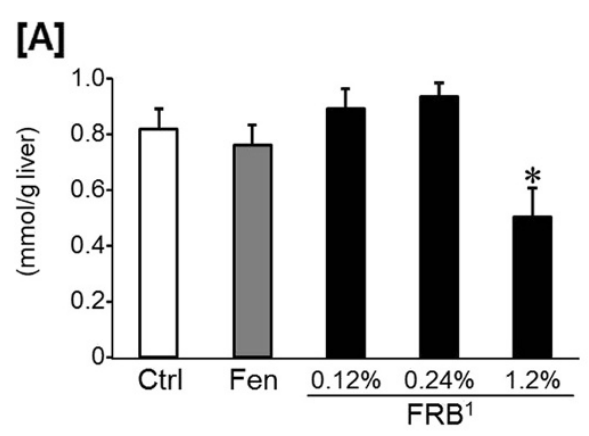

[B]

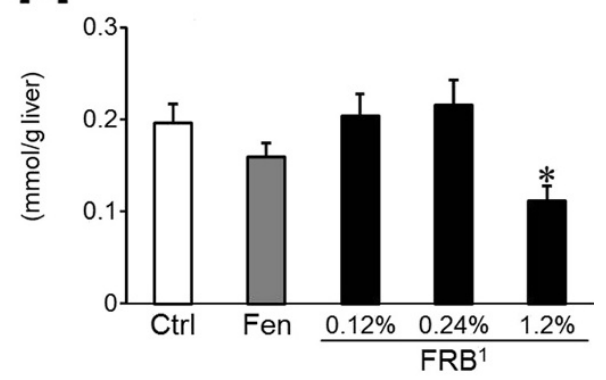

[C]

[D]
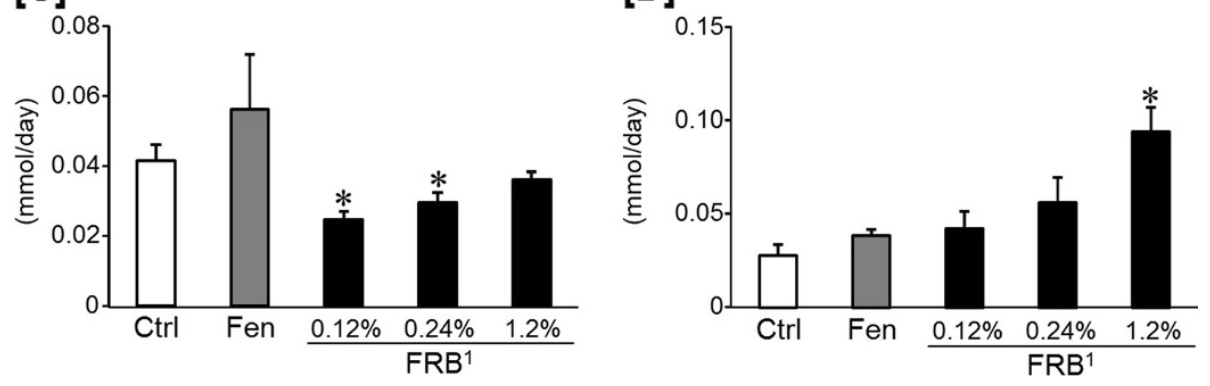

Figure 3 Effect of FRB on lipids in experiment 2. Hepatic triglyceride $[A]$ and total cholesterol $[B]$ levels. Fecal triglyceride $[C]$ and total cholesterol [D] levels. Ctrl: Control group, Fen: Fenugreek group. ${ }^{1}$ FRB contains $96 \%$ of protodioscin converted to dioscin. Values are expressed as Mean \pm SEM for $7-8$ rats. Mean without a common superscript letter is significantly different, $P<0.05$ (ANOVA). Asterisks indicate a difference from Ctrl: $P<0.05$ (t-test).

these micelles inhibit the absorption of cholesterol by directly excreting it in feces [25,26]. Diosgenin also reduces the triglyceride content and mRNA expression levels of lipogenic genes (FAS, SCD-1 and ACC) and suppresses the LXR $\alpha$ transactivation, leading to the down-regulation of both the mRNA and protein expression levels of SREBP-1c in HepG2 cells [27]. Thus, diosgenin is considered to be an active ingredient. However, a major part of protodioscin was converted by the enzyme into dioscin in the FRB. Therefore, in our study, dioscin as well as diosgenin may have contributed to the improvement of glucose and lipid metabolism.

There was a discrepancy of plasma triglyceride levels between the two experiments. We suspect that the longer period of fasting before the sacrifice in the experiment 2 might have induced the low levels of plasma triglyceride.

The results of the experiment 1 and 2 suggested that FRB had effect on lipid metabolism and glucose tolerance in the $1.2 \%, 2.4 \%$ and $4.8 \%$ FRB groups. In the experiment 1 , appetite declined significantly in the $2.4 \%$ and $4.8 \%$ FRB groups. And the soleus muscle weight and urinary creatinine levels (data not shown), which were related to the muscle mass as well as EWAT weight, decreased in these two groups. In addition, the plasma albumin and protein levels decreased in the 4.8\% FRB group as compared with the Ctrl group (data not shown). Furthermore, the plasma insulin levels were relatively high, which led to the very low level of blood glucose at $120 \mathrm{~min}$ in the $4.8 \%$ FRB group. These results suggested that overdose of FRB induced a body and muscle weight reduction, and caused a dangerous hypoglycemia.

We have demonstrated that $1.2 \%$ or more of FRB prevents diet-induced metabolic disorders including insulin resistance, dyslipidemia and fatty liver. The effective and safe dose of FRB is around $1.2 \%(\mathrm{w} / \mathrm{w})$ contained in the $\operatorname{diet}(\sim 0.25 \mathrm{~g} / \mathrm{kg} \mathrm{BW} /$ day $)$.

\section{Additional file}

Additional file 1: The English version of reference 13. Reference 13 derives from the abstract in Japanese of the original presentation. Here we translated it into English.

\section{Abbreviations}

FRB: Fenugreek with reduced bitterness; HFS: High-fat high-sucrose; EWAT: Epididymal white adipose tissue; 4-OH-lle: 4-Hydroxyisoleucine; OGTT: Oral glucose tolerance test; IPITT: Intraperitoneal insulin tolerance test; 

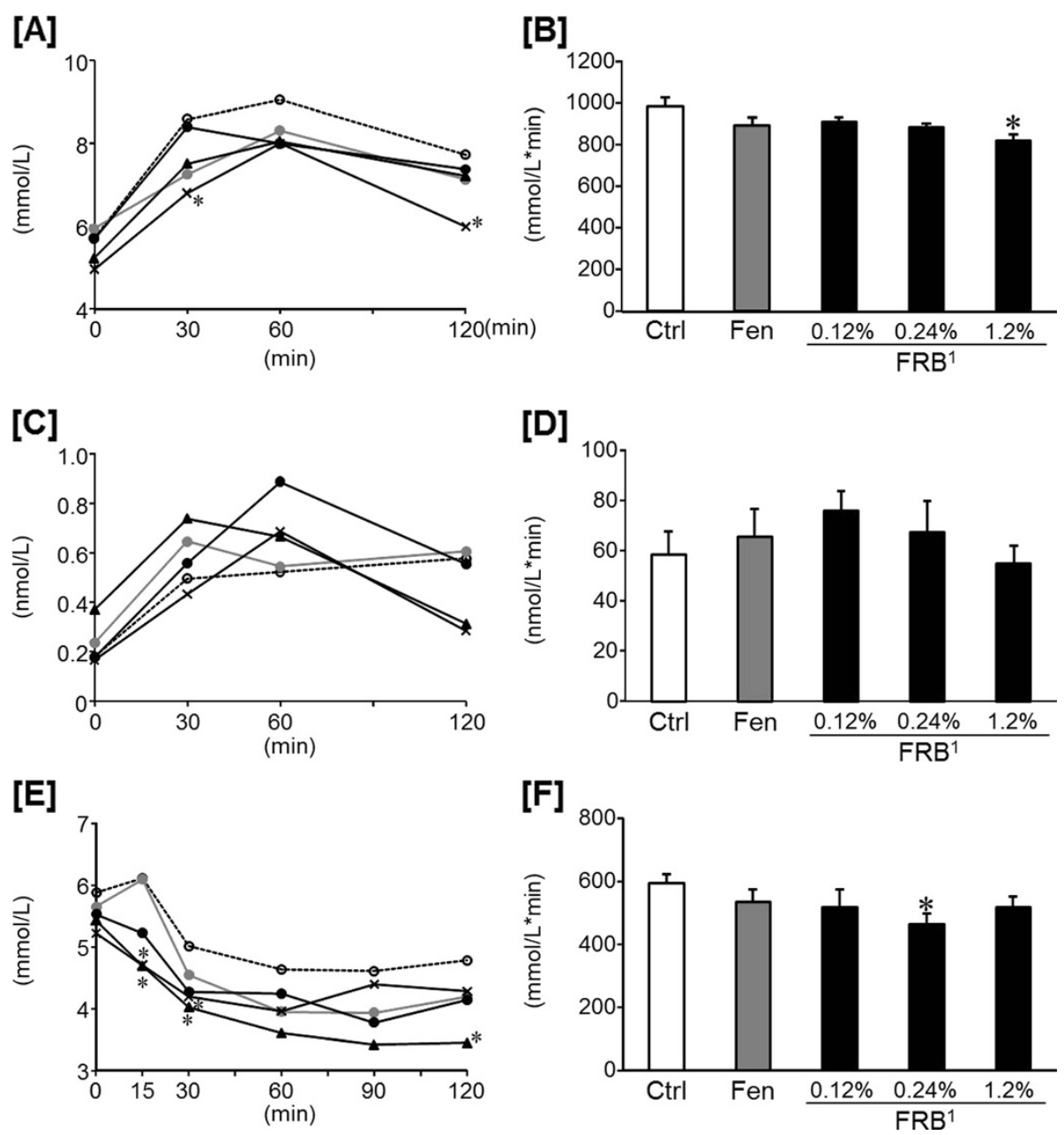

Figure 4 Effect of FRB on blood insulin and glucose levels in OGTT and IPITT in experiment 2. Changes of blood glucose [A], AUC of blood glucose [B], change of plasma insulin [C] and AUC of plasma insulin [D] after oral glucose load (2 g/ $\mathrm{kg})$. Change of blood glucose [E] and AUC of blood glucose [F] after intraperitoneal insulin load (0.75 U/kg). Ctrl: Control group (), Fen: Fenugreek group (), 0.12\% FRB group (), 0.24\% FRB group (), 1.2\% FRB group (). ${ }^{1}$ FRB contains $96 \%$ of protodioscin converted to dioscin. Values are expressed as mean for $7-8$ rats. Asterisks indicate a difference from Ctrl: $P<0.05$ (t-test).

AST: Aspartate amino transferase; ALT: Alanine transaminase; EER: Energy efficiency ratio; HOMA-IR: Homeostasis model assessment as an index of insulin resistance; AUC: Area under the curve; SDF: Water-soluble dietary fiber; IDF: Insoluble dietary fiber; PPARy: Peroxisome proliferator-activated receptor $\gamma$; MCP-1: Monocyte chemoattractant protein-1.

\section{Competing interests}

The authors declare that they have no competing interests.

\section{Acknowledgements}

We are grateful to the students Ms. Ayano Takai, Mr. Itaru Kondou, Mr. Yasuyuki Oshima and Ms. Emi Yamaguchi who assisted our study. And this study was kindly supported by House Foods Corporation.

\section{Author details}

'Department of Clinical Dietetics \& Human Nutrition, Faculty of Pharmaceutical Sciences, Josai University, Saitama 350-0295, Japan. ${ }^{2}$ House Foods Corporation, Somatech Center, Chiba 284-0033, Japan.

\section{Authors' contributions}

EM conceived the study and its design, and wrote the manuscript, carried out the experiments, and analyzed data. HC participated in the study design, assisted to carry out the experiments and draft the manuscript. $\mathrm{KT}$ and $\mathrm{SH}$ prepared fenugreek and FRB, and participated in the study design. NT and NT participated in the study design and assisted to draft the manuscript. KK conceived the study, participated in its design and helped to draft the manuscript. All authors read and approved the final manuscript.

\section{Authors' information}

${ }^{1}$ Department of Clinical Dietetics \& Human Nutrition, Faculty of

Pharmaceutical Sciences, Josai University, Saitama 350-0295, Japan.

${ }^{2}$ House Foods Corporation, Somatech Center, Chiba 284-0033, Japan.

Received: 19 February 2012 Accepted: 29 May 2012

Published: 29 May 2012

\section{References}

1. Srinivasan K: Fenugreek (Trigonella foenum-graecum): A review of health beneficial physiological effects. Food Rev Int 2006, 22:203-224. 
2. Reddy RL, Srinivasan K: Dietary fenugreek seed regresses preestablished cholesterol gallstones in mice. Can J Physiol Pharmacol 2009, 87:684-693.

3. Reddy RL, Srinivasan K: Fenugreek seeds reduce atherogenic diet-induced cholesterol gallstone formation in experimental mice. Can J Physiol Pharmacol 2009, 87:933-943.

4. Raju J, Gupta D, Rao AR, Yadava PK, Baquer NZ: Trigonellafoenum graecum (fenugreek) seed powder improves glucose homeostasis in alloxan diabetic rat tissues by reversing the altered glycolytic, gluconeogenic and lipogenic enzymes. Mol Cell Biochem 2001, 224:45-51.

5. Mohamad S, Taha A, Bamezai RN, Basir SF, Baquer NZ: Lower doses of vanadate in combination with trigonella restore altered carbohydrate metabolism and antioxidant status in alloxan-diabetic rats. Clin Chim Acto 2004, 342:105-114.

6. Gad MZ, El-Sawalhi MM, Ismail MF, El-Tanbouly ND: Biochemical study of the anti-diabetic action of the Egyptian plants Fenugreek and Balanites. Mol Cell Biochem 2006, 281:173-183.

7. Broca C, Gross R, Petit P, Sauvaire Y, Manteghetti M, Tournier M, Masiello P, Gomis R, Ribes G: 4-Hydroxyisoleucine: experimental evidence of its insulinotropic and antidiabetic properties. Am J Physiol 1999, 277:E617-E623.

8. Jette L, Harvey L, Eugeni K, Levens N: 4-Hydroxyisoleucine: a plant-derived treatment for metabolic syndrome. Curr Opin Investig Drugs 2009, 10:353-358.

9. Srichamroen A, Thomson AB, Field CJ, Basu TK: In vitro intestinal glucose uptake is inhibited by galactomannan from Canadian fenugreek seed (Trigonella foenum graecum L) in genetically lean and obese rats. Nutr Res 2009, 29:49-54.

10. Srichamroen A, Field CJ, Thomson AB, Basu TK: The modifying effects of galactomannan from Canadian-grown fenugreek (Trigonella foenumgraecum L.) on the glycemic and lipidemic status in rats. J Clin Biochem Nutr 2008, 43:167-174.

11. Muraki E, Chiba H, Tsunoda N, Kasono K: Fenugreek improves diet-induced metabolic disorders in rats. Horm Metab Res 2011, 43:950-955.

12. Muraki E, Hayashi Y, Chiba H, Tsunoda N, Kasono K: Dose-dependent effects, safety and tolerability of fenugreek in diet-induced metabolic disorders in rats. Lipids Health Dis 2011, 10:240.

13. Masamura N, Sawada H: Regarding bitter components in fenugreek (Trigonella foenum-graecum L.) seeds. In Abstract of the 14 th Annual Meeting of Japan Society for Spice Research (Nihon Koshinryo Kenkyukai koen yoshisu). 1999:22-23. cf. Preparing additional file.

14. Uemura T, Hirai S, Mizoguchi N, Goto T, Lee JY, Taketani K, Nakano Y, Shono J, Hoshino S, Tsuge N, Narukami T, Takahashi N, Kawada T: Diosgenin present in fenugreek improves glucose metabolism by promoting adipocyte differentiation and inhibiting inflammation in adipose tissues. Mol Nutr Food Res 2010, 54:1596-1608.

15. Reeves PG, Nielsen FH, Fahey GC Jr: AIN-93 purified diets for laboratory rodents: final report of the American Institute of Nutrition ad hoc writing committee on the reformulation of the AIN-76A rodent diet. J Nutr 1993, 123:1939-1951.

16. Lee SC, Rodriguez F, Storey M, Farmakalidis E, Prosky L: Determination of soluble and insoluble dietary fiber in psyllium-containing cereal products. J AOAC Int 1995, 78:724-729.

17. Bligh $E G$, Dyer $W J$ : A rapid method of total lipid extraction and purification. Can J Biochem Physiol 1959, 37:911-917.

18. Folch J, Lees M, Sloane Stanley GH: A simple method for the isolation and purification of total lipides from animal tissues. J Biol Chem 1957, 226:497-509.

19. Anderson JW, Jones AE, Riddell-Mason S: Ten different dietary fibers have significantly different effects on serum and liver lipids of cholesterol-fed rats. J Nutr 1994, 124:78-83.

20. Wong JM, de Souza R, Kendall CW, Emam A, Jenkins DJ: Colonic health: fermentation and short chain fatty acids. J Clin Gastroenterol 2006 40:235-243.

21. Comalada M, Bailón E, de Haro O, Lara-Villoslada F, Xaus J, Zarzuelo A Gálvez JM: The effects of short-chain fatty acids on colon epithelial proliferation and survival depend on the cellular phenotype. J Cancer Res Clin Oncol 2006, 132:487-497.

22. Casterline $\mathrm{J}$, Oles $\mathrm{CJ}, \mathrm{Ku}$ Y: In vitro fermentation of various food fiber fractions. J Agr Food Chem 1997, 45:2463-2467.

23. Ebihara K, Masuhara R, Kiriyama S: Major determinants of plasma glucoseflattening activity of a water-soluble dietary fiber: Effects of konjac mannan on gastric emptying and intraluminal glucose-diffusion. Nutr Rep Int 1981, 23:1145-1156.
24. Hirai S, Uemura T, Mizoguchi N, Lee JY, Taketani K, Nakano Y, Hoshino S, Tsuge N, Narukami T, Yu R, Takahashi N, Kawada T: Diosgenin attenuates inflammatory changes in the interaction between adipocytes and macrophages. Mol Nutr Food Res 2010, 54:797-804.

25. Ramadan G, El-Beih NM, Abd El-Kareem HF: Anti-metabolic syndrome and immunostimulant activities of Egyptian fenugreek seeds in diabetic/ obese and immunosuppressive rat models. Br J Nutr 2010, 23:1-10.

26. Reddy RR, Srinivasan K: Effect of dietary fenugreek seeds on biliary proteins which influence nucleation of cholesterol crystals in bile. Steroids 2011, 76:455-463.

27. Uemura T, Goto T, Kang MS, Mizoguchi N, Hirai S, Lee JY, Nakano Y, Shono J, Hoshino S, Taketani K, Tsuge N, Narukami T, Makishima M, Takahashi N, Kawada T: Diosgenin, the main aglycon of fenugreek, inhibits LXRa activity in HepG2 cells and decreases plasma and hepatic triglycerides in obese diabetic mice. J Nutr 2011, 141:17-23.

doi:10.1186/1476-511X-11-58

Cite this article as: Muraki et al:: Fenugreek with reduced bitterness prevents diet-induced metabolic disorders in rats. Lipids in Health and Disease 2012 11:58.

\section{Submit your next manuscript to BioMed Central and take full advantage of:}

- Convenient online submission

- Thorough peer review

- No space constraints or color figure charges

- Immediate publication on acceptance

- Inclusion in PubMed, CAS, Scopus and Google Scholar

- Research which is freely available for redistribution

Submit your manuscript at www.biomedcentral.com/submit
C BioMed Central 